\title{
RESUMEN DEL INFORME "UNIVERSIDAD 2000" \\ (PRIMERA PARTE)
}

\section{SUMMARY OF THE REPORT "UNIVERSIDAD 2000" \\ (PART ONE)}

\begin{abstract}
AUTORES
Juan José Ugalde Fernández: Profesor de Relaciones Públicas de la Facultad de Ciencias de la Información de la Universidad Complutense de Madrid (España). juan.jose@mixmail.com
\end{abstract}

\section{CURRÍCULUM VITAE}

Profesor de Relaciones Públicas de la Facultad de Ciencias de la Información de la Universidad Complutense de Madrid (España). Autor de varios artículos sobre la reforma de la ley de enseñanza superior en España, conferenciante y ponente en numerosos congresos universitarios y especialista en comunicación corporativa.

\section{RESUMEN}

Los servicios de educación, aprendizaje e investigación (objetivo de las universidades y, en general, de las instituciones de enseñanza superior) han de proveerse de la forma más adecuada, incorporando los nuevos procedimientos que generan las tecnologías de la información y de la comunicación, lo que obliga a revisar las formas de operar de la Universidad.

\section{PALABRAS CLAVE}

Resumen - Informes - Universidad 2000 


\section{ABSTRACT}

Education services, learning and research (target universities and, in general, institutions of higher education) have to be provided in the most appropriate, incorporating new procedures that generate the information technology and communication, making it necessary to review the modus operandi of the University.

\section{KEY WORDS}

Summary - Reports - University 2000

\section{I.- INTRODUCCIÓN: UN PERÍODO DE TRANSICIÓN EN LA UNIVERSIDAD}

1. - Cambios en la misión de la universidad.

I.- INTRODUCCIÓN: UN PERÍODO DE TRANSICIÓN EN LA UNIVERSIDAD.

1. - Cambios en la misión de la universidad.

\section{1.- Introducción.}

Los servicios de educación, aprendizaje e investigación (objetivo de las universidades $\mathrm{y}$, en general, de las instituciones de enseñanza superior) han de proveerse de la 
forma más adecuada, incorporando los nuevos procedimientos que generan las tecnologías de la información y de la comunicación, lo que obliga a revisar las formas de operar de la Universidad.

\section{2.- Una nueva transformación de la Universidad.}

Los tres grandes impactos de nuestro tiempo que han influido radicalmente sobre la demanda y sobre la provisión de la educación superior han sido: la emergencia de la sociedad de la información, que está transformando la naturaleza del trabajo y de la organización de la producción; el fenómeno de la mundialización que incide sobre las posibilidades de creación de empleo y, finalmente, la revolución científico-técnica que crea una nueva cultura y que plantea acuciantes cuestiones éticas y sociales.

Ello da lugar a que la capacitación profesional ha de permitir una continua renovación de los conocimientos para favorecer los cambios científicos y sociales y que, el aprendizaje especializado es necesario completarlo con la propia práctica profesional.

En cuanto a la demanda de investigación y al desarrollo comercial de los resultado de la misma, se configura un nuevo esquema de relaciones entre las empresas, la Administración Publica y los centros de investigación y de formación superior: es aconsejable el establecimiento de redes integradas de institutos de investigación sean o no universitarios- de consultoras o de empresas instaladas en los propios campus universitarios, relacionadas entre sí de forma flexible con el fin con el fin de favorecer sinergia entre ellas y alcanzar objetivos conjuntos de investigación.

Los cambios afectan también a los procedimientos de provisión de los servicios educativos. 
1.3.- Ampliación del número de estudiantes y diversificación de su perfil.

El crecimiento del número de estudiantes de enseñanza superior en España ha sido espectacular: mientras en el curso 1959-60 la población universitaria era de 170.602 estudiantes matriculados, en el curso 1998-99 esta cifra ascendía a 1.583.297; esta evolución ha sido común a la mayoría de los países desarrollados.

La tasa de acceso era, en 1992, del 41\% para los hombres y del 46\% para las mujeres, unas tasas de las más altas del área de la OCDE; sin embargo, las tasas de graduación eran en ese mismo año sensiblemente inferiores a los porcentajes que se dan en dicha área: del 20\% en el caso de los hombres y del 29\% en el caso de las mujeres.

Consecuencia evidente de este crecimiento es la ampliación del mercado de graduados superiores, lo que ha favorecido el crecimiento del empleo en tareas de nivel medio y superior: los titulados universitarios han pasado de representar poco más del 5\% de la población ocupada en 1976, a situarse alrededor del 17\% en 1997.

Cualquier política de futuro de la educación superior en España ha de considerar la situación y los problemas de la educación secundaria (especialmente en su tramo no obligatorio), los aspectos profesionales para los que prepara nuestro sistema de educación superior, y ha de proveer a la sociedad de formas nuevas y renovadas de enseñanzas, de nuevas formas de organizar el aprendizaje y de nuevas salidas profesionales.

\section{2.- Obstáculos a la reforma}

Son numerosas las anomalías que aquejan al sistema de enseñanza superior; pueden citarse algunas: desajustes entre el sistema de educación impartida y el número de egresados de la Universidad con relación al número de los estudiantes que ingresan 
en ella; desajustes entre los objetivos de los planes de enseñanza y las demandas que provienen del mercado de trabajo; desajustes entre los sistemas educativos nacionales y la realidad de un espacio educativo europeo.

Se suelen aducir para justificar estas anomalías las siguientes causas: la rigidez

del estatuto del profesorado; la normativa de aquellos títulos académicos que fijan condiciones de ingreso; la forma de impartir las enseñanzas; debilidad para incorporar innovaciones en las decisiones; la consideración que la opinión pública tiene sobre el mundo universitario; el riesgo político que supone tomar decisiones para transformar estructuras universitarias

3.- Algunas cuestiones de la Universidad en España

\section{1.- Los estudios.}

La incorporación de la enseñanza superior no universitaria a la Universidad, a partir de la Ley General de Educación de 1970, tuvo un doble efecto: por una parte, se uniformaron las normas y los modelos de organización de toda la enseñanza superior; por otra, la posibilidad de establecer un sistema de carreras de menor duración para la obtención de diplomas quedó, en la práctica, desvirtuado.

La L.R.U. supuso una oportunidad para adaptar la Universidad al modelo de la Universidad europea, concebida como una institución donde se conjuga la enseñanza superior y la investigación; en ciertos aspectos, la oportunidad fue aprovechada.

La reforma de los planes de estudios que introdujo se vio obstaculizada por la participación de intereses corporativos; el profesorado estuvo más atento a establecer nuevas titulaciones a partir de especialidades disciplinares y de investigación que a 
atender las necesidades que la sociedad expresaba y que ya llegaban a la Universidad: se ha tendido a uniformar las enseñanzas más que a aumentar su variedad. Aunque la citada Ley incorporó un modelo más flexible de organización curricular y se reconoció la existencia de títulos propios, éstos han acabado siendo aceptados como títulos de reconocimiento oficial.

A la demanda de estudios superiores se ha respondido con la creación de nuevas instituciones universitarias, manteniendo los sistemas restrictivos de acceso, pervirtiendo la elección inicial de los estudiantes, manteniendo el sistema tradicional del estudiante a tiempo completo; estos hechos, junto con otros, explican la elevada tasa de abandonos de los estudios y la acusada desviación entre la duración oficial o previsible de los estudios y su duración real.

Los estudios post-universitarios se han establecido, por lo general, con este carácter, a continuación de los reglados y además al margen de éstos.

Por último, se desconoce el itinerario del estudiante en su carrera universitaria y, luego, en su posterior ejercicio profesional, aunque se advierte que las probabilidades de desempleo son menores entre los diplomados en Ciencias de la Salud, seguidos por los licenciados en esta misma área, los ingenieros superiores y los licenciados en Ciencias Naturales.

\section{2.- Problemas de financiación.}

En 1995 el gasto total (público y privado) por estudiante universitario era de 4.944 dólares, mientras que la media en los países de la OCDE era de 8.134 dólares, situándose España por debajo de todos los países de la Unión Europea, excepto Grecia. 
Un 24\% de este gasto supone la aportación privada y el 76\% restante es la

aportación pública; en el resto de la Unión Europea la aportación privada es menor.

Las ayudas públicas a los estudiantes (becas y préstamos) representan el 7\% del gasto público en educación superior (el 5,4\% del gasto total), inferior al resto de los países europeos.

Además podemos destacar: la debilidad del gasto de personal docente (elevada proporción de estudiantes por enseñante: 17-1 en 1996 y bajas remuneraciones en comparación con países de la OCDE) y la alta proporción de los gastos de inversión (20\% del gasto universitario frente al 12\% de los países de la OCDE); este peso en los gastos de inversión contrasta con el de gastos corrientes, que puede tomarse como un buen indicador de la calidad de las instituciones (21\% frente al 31\% de la media de los países de la OCDE). Los programas de ayudas a los estudiantes es bastante limitada tanto en cuanto a su cobertura (en 1996-97 afectaron al 17,3\% frente al 75\% y $100 \%$ de los países nórdicos y el 20\% de los países mediterráneos) como en sus cuantías (109 ECU al mes, frente a los 200 ECU en Francia y Holanda).

Asimismo, existen diferencias apreciables en cuanto al gasto por alumno a nivel territorial: por una parte, las Comunidades con mayor gasto (Navarra, Canarias) superan en aproximadamente 200.000 pesetas a las Comunidades con menores niveles de recursos (Valencia, Galicia y Andalucía); por otra, la dispersión del gasto entre las universidades localizadas en una misma Comunidad Autónoma (las universidades catalanas mantenían en 1996 diferencias de gasto de 1 a 3,5).

\section{3.- Personal}


Tras los 17 años transcurridos desde la entrada en funcionamiento de la LRU, se ha puesto de manifiesto un diferente criterio en la aplicación de las normas en las distintas universidades en lo que se refiere a la utilización de las figuras contractuales de personal docente (Profesores Asociados, Ayudantes, aplicación de la figura del Profesor Titular de E.U.), lo que ha provocado un desequilibrio notable de la plantilla académica.

El modo de acceso a los cuerpos docentes ha sido valorado de forma negativa, atribuyéndose tal valoración a las modificaciones que la LRU introducía: excesiva endogamia, escasa movilidad del personal; sin embargo, las distorsiones en el sistema de selección son muy anteriores a la promulgación de dicha Ley.

Existe una disparidad en las plantillas docentes entre universidades, apreciándose por la diversidad en el porcentaje de doctores: 42\% en 1991, 43\% en 1995; por debajo de este porcentaje se sitúan las universidades nuevas, las politécnicas y las que han descentralizado sus campus.

El P.A.S. es un personal de apoyo básico e imprescindible para las tareas fundamentales de la Universidad; está aumentando su importancia y está adoptando nuevos roles y técnicas de trabajo más especializadas. Aunque se rige por la legislación básica de funcionarios (el personal funcionario) o por el Estatuto de los Trabajadores (el personal laboral), la propia autonomía universitaria, al amparo de su potestad de auto-organización, permite importantes modulaciones y especialidades, destacando la pluralidad y la variedad de sus componentes.

La dispersión existente en el régimen del P.A.S. en las distintas universidades ha dado lugar a un sistema universitario cuyo personal docente presenta perfiles comunes y un P.A.S. que posee un estatuto diferenciado en cada universidad, tanto a nivel retributivo, promoción, movilidad, etc. 


\section{4.- Investigación.}

Constituye una actividad relativamente reciente que consagró la LRU, al establecer la doble dimensión en las universidades como centros docentes y domo centros de investigación; la citada Ley introdujo incentivos a la realización de I+D con contrato o en colaboración con el sector privado, sin embargo, la investigación en la universidad ha sido financiada mayoritariamente por las Administraciones Públicas. El gasto de I+D ha pasado de 25.000 millones de pesetas en 1983 a 219.951 en 1997; el número de investigadores ha crecido de 13.033 a 30.649 en el mismo período. Ahora bien, este gasto es muy inferior a la de la mayoría de los países más avanzados en términos de participación en el PIB (0,86 en 1997 frente al 2,2\% de la media de la OCDE).

En el sistema de I+D hay que distinguir entre la participación pública y la privada, tanto desde la perspectiva de la financiación como de la ejecución del gasto: en 1995 el 52,4\% corresponde a las Administraciones Públicas y el $40,3 \%$ a las empresas privadas, frente a la media de la OCDE del 33,8\% y 59,9\%, respectivamente, lo que pone de manifiesto el bajo esfuerzo en I+D que realizan las empresas privadas en relación con los países de la OCDE. Además, la distribución territorial del gasto del sector empresarial español es muy desigual: en 1997 el 76,8\% del gasto ejecutado se concentró en tres comunidades autónomas: Madrid (34,5\%), Cataluña $(28,1 \%)$ y el País Vasco (14,2\%); ahora bien, los porcentajes de la parte de la I+D ejecutada por las universidades de estas mismas tres comunidades autónomas son bastante inferiores a la media del conjunto del Estado.

\section{II.- UNIVERSIDAD, AGENTE SOCIAL.}

1.- Una incesante adaptación a las funciones. 
1.1.- La Universidad es una institución autónoma que, de manera crítica, produce y transmite la cultura por medio de la investigación y de la enseñanza; ya desde principios del siglo XIX se advierte que los Estados tienden a influir sobre ellas en una determinada dirección.

La universidad constituye un medio adecuado para conseguir determinados fines de carácter colectivo. Nos referimos a:

elaboración y transmisión de los resultados de la reflexión y la investigación a través de una institución a la que sus miembros deciden integrarse de forma voluntaria.

existencia de una corporación.

independencia en su funcionamiento respecto de los poderes exteriores a la misma.

capacidad de asegurar la continuidad de su actividad en el tiempo.

1.2.- La corriente Humanista no fue acogida en la Baja Edad Media. Las universidades europeas no aceptaron la filosofía y la literatura que promovía el humanismo hasta el siglo XVI.

Con la revolución científica a finales del siglo XVIII aparecen las primeras universidades técnicas y la enseñanza superior para responder a las nuevas demandas técnicas y sociales fomentó su generalización.

La investigación asegura el desarrollo de la creatividad y acostumbra a los estudiantes a cuestionarse los logros científicos y los saberes adquiridos. 
2.- Conocimiento, innovación y aprendizaje.

2.1.- Un proceso histórico.

3.- La mayor parte de la actividad humana en las sociedades, en el siglo XVIII, se centraba en los procesos de control de los medios de producción. El desarrollo económico ha aumentado y ha estimulado la expansión de la demanda de servicios, ha convertido la formación en un factor fundamental.

La sociedad se reorganiza para afrontar los procesos sociales y económicos, para lo que necesita desarrollar el conocimiento humano.

4.- Las sociedades industriales han encabezado el desarrollo mundial, especialmente desde los años 70, la capacidad de aprender es el fundamento principal de las sociedades modernas. Conocimiento, innovación y capacidad de aprendizaje son los pilares de las sociedades avanzadas.

2.2.- Cuatro dimensiones de un cambio estructural.

5.-

la generación de nuevos avances científicos y las TIC.

el reparto de la actividad económica.

la internacionalización de las sociedades y sus economías.

el aumento del nivel de educación.

6.- La capacidad de difusión de las TIC deben dar lugar a las innovaciones organizativas para poder aprovechar las ventajas sociales asociadas a los avances tecnológicos. 
7.- El reparto de la actividad económica y los cambios en el funcionamiento de los mercados.

8.- La internacionalización de las economías, compromisos entre empresas en el ámbito de la I+D que sirve de soporte a los flujos de capital y a los activos de conocimiento.

9.- Los procesos de formación y aprendizaje.

10.- Las importantes inversiones publicas y privadas en I+D reflejan el exponente de la capacidad de innovación de un país.

11.- Las inversiones en TIC y poder realizar importantes cambios organizativos.

12.- Inversiones destinadas a formación y adquisición de nuevas cualificaciones.

2.3.- Papel central de los procesos de innovación.

13.- La competencia tecnológica.

14.- Eje de las políticas económicas.

15.- Compensar la insuficiente provisión privada en I+D.

16.- Gestión financiera apropiada.

17.- Innovación como proceso interactivo.

18.- Capital social y capital humano. 
19.- Procesos de aprendizaje y adaptación.

20.- Acceso a conocimientos.

21.- Capacidad de absorción.

22.- Vías a nuevos conocimientos.

2.4.- Las nuevas referencias de la política social y económica.

23.- Mayor interdependencia de las economías nacionales.

Capacidad de las empresas para competir en mercados.

24.- Integración coherente.

25.- Política de fomento de la ciencia y del desarrollo tecnológico.

Política de educación y aprendizaje.

26.- Relaciones entre la ciencia y la innovación.

27.-

Potenciación y mejora de las relaciones entre instituciones públicas.

Fomento público de la investigación básica.

Potenciar la política de transferencia tecnológica y difusión de los resultados.

Desarrollar una política de bienes colectivos. 
Fomento y apoyo a una capacidad empresarial.

Consecuencias de la inversión internacional.

28.- Validación y difusión de nuevos conocimientos.

29.- Preservar y transmitir los valores sociales.

30.- Se deberían introducir materias humanísticas de manera transversal para tratar de acortar distancias entre la llamada cultura humanística y la cultura científica.

3.- Universidad y entorno social.

3.1.- Cultura y consumo.

31.- Organización social, producción/consumo.

32.- Habilidades de producción y consumo.

33.- Orientación hacia nuevos campos en colaboración con otras organizaciones de carácter local y de niveles superiores.

34.- Integración de representantes de las entidades más significativas en sus órganos internos.

3.3.- Generalización de la educación superior: el acceso a la universidad.

35.- Criterios de asignación:

Haber superado las PAAU.

Nota de acceso + expediente académico. 
Oferta/demanda.

36.-

Exceso de demanda en:

Areas de Ciencias de la Salud y Enseñanzas Técnicas.

Ciencias Sociales y Jurídicas.

Demanda estancada en:

Ciencias Experimentales y Humanidades.

Segundas demandas:

Ciencias Sociales, Jurídicas, Experimentales y Humanidades.

37.- Vías de ingreso:

Procedentes del Bachillerato.

Formación Profesional.

Pruebas mayores de 25.

38.- Fenómeno oferta/demanda.

Mayor oferta de plazas.

Mayor información y orientación.

Actuación sobre la oferta.

Coordinación territorial.

Sistema de distrito.

39.- La mundialización de la sociedad hacia un espacio europeo.

Criterios estrictamente académicos.

Autonomía universitaria.

3.4.- Dimensión territorial de las universidades. 
40.- Presiones de los poderes públicos y de la sociedad en la mayoría de los países de la OCDE.

41.- Intercambios entre la universidad y la industria y en general con su entorno.

42.- Aportar la aparición de líderes de la sociedad política y civil, así como creadores de opinión.

43.- Decisiones de inversión y atracción de nuevas actividades.

44.- Estimular la creatividad y la innovación, garantizar las capacidades de producción y consumo y asegurar conexiones con el resto del mundo.

45.- Son piezas esenciales en la generación y el mantenimiento de las capacidades.

46.-Establecer el diálogo con los demás agentes sociales del propio territorio.

47.- Necesidad de cambios:

Excesiva cultura académica.

Incentivos para una mayor apertura al entorno local.

Reformar las propias estructuras sociales.

4.- Instituciones de la política universitaria.

Los gobiernos se plantean como objetivos:

Una mayor preparación de técnicos para favorecer la innovación y la competitividad. 
Garantía e igualdad de oportunidades de acceso.

Transmisión de los valores culturales como cauce para el progreso.

La descentralización y la autonomía son el marco más idóneo para organizar las enseñanzas de nivel superior y para rendir cuentas ante la sociedad en general y a las Administraciones Públicas en particular.

Sería igualmente importante establecer unos criterios de delimitación de las competencias sobre investigación y enseñanza superior. También sedería crearse un Consejo que actuase como órgano coordinador de las distintas Administraciones.

\section{III.- DIFUSIÓN DEL CONOCIMIENTO: FORMAR PARA APRENDER}

El capítulo comienza con una introducción referida a la necesidad de adaptación de la educación a nuevas exigencias y remarca dos hechos de especial importancia como son la consolidación de un sector no universitario de enseñanza superior; y la creciente percepción social de que la formación superior ha de acompañar a las personas a lo largo de su actividad laboral. De aquí la importancia de la formación continuada no sólo por si, sino por lo que implica de diversificación en la propia enseñanza superior y en el mismo seno de la Universidad, donde su encaje pone en cuestión algunas estructuras de la universidad actual, al quebrar la división disciplinar de la misma y demandar nuevas formas específicas de organización. Respecto de estas últimas el Informe apunta que en el seno de cada universidad, la estructura responsable de los programas de formación continuada puede adquirir formas centralizadas, formas descentralizadas o bien formas autónomas de organización de las mismas, es decir, en el primer caso se plantea como un servicio con trazos propios, dependiente de los servicios centrales de la universidad; en el segundo caso son las Facultades o departamentos quienes gestionan de manera independiente los programas; y finalmente, en el tercer caso se confían estos a una entidad jurídica diferenciada como puede ser el caso de una Fundación... etc. 
A continuación el Informe entra a especificar cuál debiera ser el papel que han de desempeñar las administraciones públicas, teniendo en cuenta las crecientes tasas de difusión de la enseñanza superior en las sociedades desarrolladas, lo cual exige de las universidades mayor flexibilidad e innovación, partiendo de un enfoque centrado en el propio estudiante, el cual a su vez, presenta un perfil diferente al que venía siendo habitual.

En este sentido se apuesta por circunscribir el papel de las administraciones a:

Determinar las prioridades educativas

Garantizar, mediante sistemas de evaluación externa, unos niveles mínimos de calidad para cada institución y enseñanza, de acuerdo con los objetivos que ellas mismas se marquen.

Verificar la idoneidad de los estudios ya emprendidos por las instituciones con el fin de obtener determinadas cualificaciones académicas y profesionales.

Asegurar la financiación suficiente si se cumple con lo anterior y se gestiona correctamente.

Organizar un sistema adecuado de información a los estudiantes.

Precisar el modo de introducir transparencia en los estudios realizados y fomentar la movilidad de los estudiantes, fijando el crédito como unidad de aprendizaje y determinando claramente la significación de las distintas titulaciones y grados.

Dotar a las instituciones de enseñanza superior de suficiente autonomía cuidando de que asuman su responsabilidad ante la sociedad.

Es decir se aboga por un nuevo esquema de relaciones entre las administraciones y las instituciones académicas basado en mecanismos de acreditación a posteriori y en principios de mutua transparencia. 
El Informe aborda a continuación la estructura de las enseñanzas, partiendo de la distinción entre los estudios en los que el elemento decisivo y unificador son los factores disciplinares de formación que se revelan en la enseñanza y en los programas científicamente fundamentados (los que tradicionalmente han impartido las universidades, y los estudios profesionales en los que el énfasis se pone en las competencias adquiridas.

Se propone la siguiente clasificación:

Estudios de tipo A, de carácter disciplinar. Tendrían cuatro años de duración y, a su vez, la dividirían en dos tramos: un primer tramo de carácter inicial, de dos años como mínimo, de tipo pluridisciplinar en buena medida, común a las grandes áreas científicas(ciencias, humanidades, ciencias sociales, ciencias de la vida, ingenierías) y un segundo tramo de carácter especializado o más monodisciplinar.

Estudios de carácter profesional de tipo B, con programas integrados de tres años de duración. Estos estudios acogerían los equivalentes a los antiguos peritajes, estudios de enfermería, magisterio, etc. Así como estudios impartidos de forma aislada propios de una formación profesional de carácter superior.

El segundo grado estaría conformado por:

Estudios disciplinares de tipo $C$, en los que se pretende una ulterior profundización disciplinar que, en muchos países otorga el título de master o magister y que corresponde a un postgrado en una determinada disciplina. Se cursarían durante uno o a lo sumo dos años.

Estudios profesionales de tipo D que se cursarían también durante uno o dos años y que acogerían estudios integrados como en el caso de las ingenierías y estudios más dispersos como los que conducen a la obtención del título de "master" en su actual acepción y práctica española, donde domina la referencia profesional. 
El tercer grado de formación sería para el doctorado, cuyos estudios se organizarían en un solo tramo que conduciría a una sola clase de doctorando, tras superar los estudios de tipo C o de tipo D suficientemente integrados.

Los estudios de carácter técnico, que en España se denominan ingenierías, podrían organizarse de manera alternativa mediante una primera fase de tipo A, que permitiría acceder a una segunda fase de tipo $\mathrm{D}$, $\mathrm{u}$ otra única de tipo B.

Igualmente se propone que junto a las titulaciones que definen el grado alcanzado en la superación de laos diferentes ciclos de la enseñanza superior, se reconozca mediante las oportunas certificaciones los cursos aislados de tipo B y D y el tramo pluridisciplinar de tipo A con el fin de informar sobre los distintos estudios realizados, en le caso de que estos no permitan alcanzar titulación alguna por interrupción o abandono de los mismos.

Esta estructura de estudios debe permitir a los estudiantes elaborar su propio itinerario curricular, en el marco de un número preciso y reducido de condiciones. Ello exige la conversión de los estudios al esquema de créditos (ECTS) "European Credit Transfer System" que mide las enseñanzas recibidas en términos de clases, trabajos prácticos, seminarios y exámenes u otros métodos de evaluación. Este procedimiento facilita la movilidad entre las diversas enseñanzas y el reconocimiento académico entre instituciones a través de la consideración de que los créditos son de aplicación general y se basan en el principio de que, para toda institución de educación superior, un año académico equivale a una cantidad fija de 60 créditos.

El Informe apunta también muchas otras sugerencias entre las que destacan las referidas a que en los distintos cursos y programa debiera figurar la conveniencia de seguir estudios en otras universidades (tras haber cursado los primeros cursos) así 
como detallar las actividades que desarrollar en centros de trabajo, las cuales deberían ser tuteladas conjuntamente.

Igualmente merece destacarse la idea de que la organización de los cursos de tipo A y C podría atribuirse generalmente a los departamentos mientras que los cursos de tipo B y D se deberían organizar según sistemas alternativos. En los mismos, los responsables de estos cursos deberían dar cuenta de su enfoque ante los organismos centrales de las instituciones y para llevarlos a cabo se debería contar con la colaboración de personas ajenas a la Universidad.

La determinación del marco general y de los mínimos curriculares de los programas debería corresponder a las administraciones públicas, quienes deberían limitarse a estos mínimos excepto para aquellas profesiones para cuyo ejercicio exista una tradición, compartida por diferentes países, de regulación profesional.

Dichos criterios mínimos deberían abarcar los grandes campos del conocimiento, campos sin duda más amplios que las actuales titulaciones correspondientes a cada grado equivalente a una licenciatura o a una diplomatura.

Así mismo las Administraciones públicas, en el ámbito de sus competencias territoriales, deberían establecer, con carácter plurianual, las distintas opciones de estudios preferentes que desean que se lleven a cabo. La delimitación de estas opciones tendría el carácter de directivas con efectos positivos para la financiación de dichas instituciones a través de los contratos-programa.

Las universidades y demás instituciones de enseñanza superior deberían formular sus propuestas de estudios atendiendo a sus características. 
Los planes de estudio deberán ser transparentes y han de facilitar la máxima información y cuando conduzcan a títulos académicos, la institución que los promueva debería someter su propuesta a las Administraciones públicas competentes, las cuales podrían oponerse a dicha propuesta, dentro de un plazo determinado, sin requerirse una aprobación formal, la cual se entendería concedida cuando hubiera transcurrido dicho plazo, sin formular oposición expresa.

Esta aprobación por parte de la Administración tendría carácter provisional, hasta que la institución implicada no recibiera la acreditación posterior sobre la idoneidad de su programa y obtuviera, una evaluación suficiente sobre la calidad del mismo. Esta acreditación deberá renovarse en sucesivas ocasiones.

El Informe culmina este capítulo con una serie de sugerencias encaminadas a facilitar apoyo, información y asesoramiento al estudiante. Promueve igualmente que la enseñanza se apoye en el uso de las modernas tecnologías y apunta la necesidad de corregir la notoria disparidad entre la duración real de los estudios y su duración oficial, para lo que propicia corregir las situaciones de "rigor desproporcionado" y atribuir un número especial de créditos (equivalentes a los normalmente necesarios para conseguir uno de los grados) a cada estudiante el cual los utilizaría en su matrícula y una vez consumidos terminaría su derecho a proseguir sus estudios.

\section{VALORACIÓN}

En general este capítulo es de los más precisos y responde positivamente a la necesidad de un adecuado encadenamiento de los diferentes estudios, haciéndolo compatible con una notable capacidad de elección del alumno.

Favorece la tendencia hacia la armonización del espacio europeo de educación superior e integra la componente profesional como complemento a la disciplina para 
el conjunto de las titulaciones, lo cual permite una mejor acomodación de las exigencias del sistema productivo sin que se vea mermado el carácter de una formación universitaria que no puede responder únicamente a esa finalidad.

Igualmente es respetuoso con la autonomía universitaria y le concede a las administraciones públicas el papel de supervisores de la necesaria calidad del sistema al tiempo que le sugiere vías para facilitar que los intereses generales de la sociedad sean atendidos.

En general parece muy adecuado como respuesta a los problemas que en este ámbito están planteados.

\section{IV.- GENERACIÓN Y APLICACIONES DEL CONOCIMIENTO.}

Las principales propuestas, se recogen en siete líneas básicas de actuación.

1. Aumento del gasto en ciencia y tecnología, hasta alcanzar el promedio de la UE. Aunque se resalta el hecho de que sólo la $1 / 2$ del gasto en I+D corresponde al sector privado, frente al $2 / 3$ privado y $1 / 3$ público de la UE. No se aporta ninguna propuesta para superar este claro desequilibrio, como no sea el aumento de personal en ambos sectores y la obtención de fondos de la UE.

2. Potenciación y mejora de las relaciones entre la universidad y el sector empresarial.

Se propone una participación mas activa de las universidades en el proceso innovador. Entre las que destacaría la creación de empresas y el reconocimiento y valoración de los parámetros de innovación (patentes, asistencia técnica,...). 
Además se apuesta por consolidar centros de investigación tanto en ciencias básicas y aplicadas como de humanidades, como medio de reforzar la implicación del sector universitario en los procesos de innovación.

\section{El fomento de la investigación básica.}

Este apartado al que se dedica una mayor extensión, presenta propuestas que recomiendan un aumento de fondos en las actividades de investigación básica, así como favorecer iniciativas que aumenten su excelencia y calidad.

\section{Potenciación de la política de transferencia tecnológica.}

La participación de las universidades en dos tipos de iniciativas como son los consorcios administración-empresas de un determinado sector productivo y las asociaciones de empresas de idéntica y complementaria actividad.

5. Apoyo de nuevas iniciativas empresariales asociadas a las nuevas tecnologías.

Este apartado básicamente repite lo ya expuesto en la línea de actuación $2^{\mathrm{a}}$. Creación de empresas por los propios investigadores, movilidad de los investigadores con las empresas, reconocimiento de parámetros de innovación (patentes, asistencia técnica..)

6. Mayor difusión territorial del esfuerzo en I+D y de innovación.

Creación de una red de centros que sirvan de apoyo a los centros de investigación y a las empresas.

7. Potenciación de los esfuerzos en formación de nuevos investigadores.

Se sugieren modificaciones en los estudios del 3er ciclo de manera que se prestigie el título de doctor. 
Estas son, a modo de resumen, las principales líneas de actuación que el informe Bricall propone para la investigación en la Universidad.

\section{VALORACIÓN.}

La investigación en la universidad presenta una peculiaridad, si la comparamos con el resto de los organismos públicos de investigación, (CSIC, INIA, ..). Esta peculiaridad le viene conferida por el hecho de ser sólo un grupo de profesores los que desarrollan de forma continuada proyectos de investigación, probablemente estos representan el $40 \%$ de todo el profesorado, variando con la Universidad y los Departamentos.

Aquellos profesores que no desarrollan investigación pueden dividirse en dos grupos.

a) Los que por el número de clases y una falta de medios (doctores capaces de dirigir las tesis) se encuentran condenados a no realizar investigación alguna.

b) los que siendo funcionarios no consideran a la investigación como una actividad complementaria de la actividad docente.

Esta introducción es imprescindible a la hora de plantear análisis y entender la acción sindical sobre la investigación en la universidad.

Para los que intervinimos en las jornadas que han dado lugar al informe Bricall, estaba claro que nos estabamos refiriendo a aquellos grupos de profesores implicados de forma continuada en proyectos de investigación, los otros grupos requieren otros planteamientos que escapan a los que a continuación exponemos. 
En su conjunto el informe representa un intento importante de análisis y de ofrecer propuestas a la investigación universitaria, considero que estas no contradicen nuestras propuestas en investigación, aunque significaran cambios que no sabemos si serán entendidos por el conjunto del profesorado.

Nuestro sindicato debe hacer un esfuerzo y discutir seriamente las propuestas que se contemplan en el informe, y no sólo desde la perspectiva del profesor dedicado a la docencia, sino desde la perspectiva del profesor/investigador implicado en el desarrollo de proyectos de investigación en una universidad donde la actividad docente, aunque mal valorada, es su razón de ser.

Al margen de una valoración global positiva quiero resaltar algunas cautelas que deben tenerse en cuenta sobre las propuestas anteriormente reseñadas.

El informe no trata de las precarias condiciones de trabajo de los investigadores universitarios.

Deberemos apostar por la eliminación de las becas como forma de relación contractual entre los investigadores y los proyectos. Las becas deberán quedar reducidas exclusivamente al periodo de formación y ser sustituidas posteriormente por contratos de larga duración

La creación de la figura del investigador de plantilla en las Universidades, propuesta por el informe deberá abrir un debate sobre sus actividades.

Frente a un apoyo desmesurado a las grandes concentraciones de científicos en Institutos, Centros de investigación,........ Proponemos el que no se olvide el apoyo de la investigación realizada en grupos pequeños, unidos a la existencia de unos servicios centralizados de apoyo a estos grupos. 
En definitiva solicitamos el aumento de la masa crítica de investigadores en todo el territorio nacional y no sólo en Madrid y Cataluña como sucederá si apoyamos a los grandes grupos, además el aumento de investigadores garantizará que la ciencia realizada en las universidades responda a lo que en realidad queremos que sea, un servicio público de interés social.

La introducción de nuevas figuras como las empresas universitarias, los consorcios y los parques tecnológicos, deben de ir acompañadas de unos mecanismos transparentes de control y de un papel más activo por parte de los sindicatos a la hora de vigilar los tipos de contratos y de relaciones contractuales que se establezcan entre la universidad y los investigadores/trabajadores de estas empresas.

Solicitar la participación de las fuerzas sociales (sindicatos y empresarios) en el control de los planes de investigación. Recientemente en el III Plan Andaluz de Investigación se ha conseguido introducir una comisión de control surgida del Consejo Asesor de Ciencia y Tecnología.///

\section{V.- FINANCIACIÓN}

1- Introducción

1. Objetivos de un posible sistema de financiación: a) mejorar la eficiencia y la calidad de la enseñanza superior; b) lograr una mayor igualdad de oportunidades.

2. Las universidades se financian con tres tipos de fondos: tasas (precios), subvenciones y otros.(Véase apartado I-34). Las tendencias OCDE son hacia un aumento de la financiación privada frente a las subvenciones públicas que siguen manteniendo una presencia mayoritaria. El aumento de las tasas exige incrementar 
los préstamos-renta y las becas, para garantizar la equidad e igualdad de oportunidades.

3. Aspectos que diferencian los diferentes modelos de financiación:

Importancia atribuida a las tasas (uniformes, dos partes y bloques).

Oferta de becas

Políticas para obtener "otros ingresos"

Alcance y características de las subvenciones públicas

4. La combinación óptima debe perseguir los siguientes objetivos:

Suficiencia financiera

Eficiencia interna

Eficiencia externa

Igualdad de oportunidades

5. Plantea la necesidad de combinar adecuadamente los procedimientos de obtención de recursos

2.- Financiación privada y financiación pública a los estudiantes

2.1. Tasas académicas y ayudas al estudiante

6. En la OCDE las tasas suponen entre el 0 y el $20 \%$ del coste total de los estudios.

7. Durante la última década se ha producido un incremento de las tasas al mismo tiempo que se ha procedido a la reforma de las políticas de ayudas al estudiante. 
8. No se ha comprobado una relación clara entre el importe de las tasas y la tasa de escolarización, no obstante se observa que las familias de rentas bajas responden mucho más a cambios en los precios o en el coste neto de los estudios.

9. Se argumenta contra la gratuidad total de la enseñanza universitaria.

10. "La gratuidad de la enseñanza no basta para alcanzar la igualdad de oportunidades."

11. La igualdad de oportunidades no puede alcanzarse únicamente mediante becas o supresión total de tasas. Hay que proceder a reformas tendentes a la evaluación docente, revisión de planes de estudio y normas de permanencia. La mejora de la eficiencia requiere modificaciones de la gestión interna y de los órganos de gobierno. "La financiación pública de la enseñanza superior podría ser regresiva".

12. Se deben combinar tasas, becas y programas de préstamos.

13. Para garantizar la igualdad de oportunidades es necesario establecer un programa de préstamos garantizados por el Estado.

14. El sistema de préstamos es más eficiente, porque incentiva el esfuerzo del alumno; y es más equitativo, ya que elimina la dependencia respecto a las familias.

15. Los préstamos pueden desincentivar a familias de bajo nivel socioeconómico. Para evitarlo hay que combinar préstamos y becas y acudir al préstamo-renta.

16. Analiza una propuesta teórica de implantar el impuesto universitario, que ha tenido un cierto eco entre los expertos pero no ha llegado a aplicarse en ningún país. 
17. Constata que la contribución de los usuarios al sostenimiento de las universidades públicas está creciendo en el conjunto de la OCDE.

18. Analiza la situación y evolución de tasas, becas y préstamos en varios países, especialmente de Australia, Nueva Zelanda y Reino Unido

19. El sistema español de financiación no ha sido capaz de combinar tasas y ayudas. Hay una escasa cobertura de ayudas a los estudiantes. Se recomienda utilizar prioritariamente las becas en los primeros años y los préstamos-renta en los ciclos posteriores.

\subsection{Becas}

20 Estudia los tipos de becas y el grado de cobertura de las mismas en diversos países, haciendo especial mención de Estados Unidos, Australia, Nueva Zelanda y Holanda.

21. En España el sistema de becas es insuficiente y además no es siempre progresivo, debido al fraude fiscal y a la combinación de criterios académicos y económicos.

22. Los requisitos académicos llevan a estudiantes de renta más baja a perder su beca. Se sugiere reducir o eliminar el peso de los criterios académicos. Esta reforma debe ir acompañada de una revisión de las normas de permanencia (Véase III-63).

23. Otra limitación importante reside en el importe de las ayudas. Sería deseable que la cuantía de las becas compensara el coste de oportunidad de estudiar.

24. Las ayudas por cambio de residencia son de escaso importe. Se debe promover la movilidad con un sistema de becas no discriminatorio (Véase II-39 y V-33). 
2.4 Préstamos-renta (es evidente que hay un error en la numeración. Debería ser el 2.3)

25. Los préstamos se pueden utilizar para financiar:

Los costes directos de la enseñanza (tasas o precios).

Los costes indirectos (alojamiento, transporte, libros, etc.).

26. Para estudiar los créditos hay que tener en cuenta las siguientes cuestiones:

Elegibilidad

Forma y cuantía de las subvenciones

Carácter público o privado de los fondos

Condiciones de su devolución

27. Elegibilidad: Las instituciones de crédito no han de tener capacidad para seleccionar. Las Administraciones Públicas son las únicas que han de decidir quién tiene acceso. En general suelen tenerse en cuenta dos criterios: la renta familiar y los resultados académicos. En muchos países la concesión tiende a ser prácticamente universal.

28. Los préstamos suelen incluir una componente implícita de ayuda pública, ya sea en forma de tipo de interés, período de carencia o de un aval público.

29. Los fondos prestados son a menudo públicos. En otros casos, son privados, pero con garantía del Estado. Debe existir un control público de devoluciones.

30. Los préstamos se pueden devolver en plazo fijo o en función de la renta.

31. Riesgo de falencia. Medidas a tener en cuenta: 
Posibilidad de largos períodos de devolución

Sistema flexible de amortización

Control de los prestatarios a través de alguna instancia pública

32. Los programas de préstamos con devolución condicionada según la renta parecen resolver la mayoría de los problemas.

33. Parece adecuado empezar con un programa experimental de préstamos-renta, priorizando modelos que faciliten la movilidad.

34. Configuración de los préstamos-renta:

ELEGIBILIDAD: restringidos a estudiantes de los últimos cursos. Las condiciones deben ser controladas siempre por la Administración Pública.

CUANTÍA DEL PRÉSTAMO: en torno a 600.000 ptas./año por estudiante.

TIPO DE INTERES Y CARENCIA: tipo de interés nulo o muy reducido. Se comenzarían a devolver al superar la renta per cápita media del país.

DEVOLUCIÓN: deben detallarse los períodos y las cuotas.

35. En la mayoría de los casos es la agencia tributaria quién controla el proceso de devolución de los préstamos.

3.- Financiación pública directa a las instituciones de enseñanza superior

36. La subvención pública es la principal fuente de financiación de la enseñanza pública. Las subvenciones públicas directas no han respondido a un modelo que relacionase los recursos y los objetivos. Los fondos repartidos lo han sido en función de años anteriores - modelo incrementalista -. En épocas más recientes la introducción de esquemas de incentivos favoreció la eficiencia y la calidad. El sistema incrementalista tiene problemas de descrédito o busca objetivos políticos. 
Considerar la sustitución del modelo incrementalista y burocrático por uno basado en objetivos educativos. Modelos de este tipo se utilizan ya en la OCDE.

37. Las fórmulas de financiación en Estados Unidos, desde Tejas años cuarenta, cuentan con ventajas como garantías de trato similar y mismas reglas del juego para todas las instituciones, transparencia y flexibilidad. El elemento innovador en los años sesenta de presupuestación en base cero mejora la eficiencia económica, aunque genera incertidumbre y requiere gran información sobre costes. En los años noventa aparece la tendencia de ligar la financiación a indicadores de rendimiento. Destacar que en los años ochenta se exigió que se evaluase el aprendizaje, se controlase la actividad docente y la dedicación a la enseñanza ya que existía la sospecha de que se privilegiaba la investigación por encima de la enseñanza. El gobierno federal exigió establecer agencias de información y evaluación de la enseñanza superior. En la Unión Europea han destacado los modelos y fórmulas de Reino Unido y Holanda.

En el Reino Unido en los años ochenta se modificó la financiación pública aplicando nuevas fórmulas que han reducido la financiación por estudiante. En conclusión, el Reino Unido utiliza financiación mediante fórmulas con imputs (estudiantes) y outputs (investigación).

En los países bajos hay cuatro tipos de modelos con tendencia a ligar la financiación a los outputs, planea sustituir la fórmula por un contrato de 5 a 10 años dando un horizonte estable a los centros.

Francia ofrece un modelo centralizado. En 1976 establece una fórmula de financiación basada en la carga docente y el coste de la enseñanza. Se introduce una nueva fórmula en los ochenta basada en costes estándar por estudiante. En los noventa se reforma introduciendo contratos programa.

Finlandia usa el modelo contractual.

Nueva Zelanda usa contratos-programa. 
Australia, en 1974, suprimió las tasas académicas. En 1989 reforma con una combinación HECS, aumentando la autonomía y exigiendo una precisa rendición de cuentas.

Dinamarca establece en 1992 una financiación en función del output estudiantes aprobados, promoviendo la titulación y con posibles efectos perjudiciales sobre la calidad evitándolo con mecanismos de control de calidad.

38. En resumen, pueden diferenciarse las siguientes características:

Subvención calculada a partir de imputs y outputs.

Fondos asignados a criterios objetivos o gastos justificados.

Financiación para usos específicos o libertad en su asignación.

39. En general, los sistemas tienden hacia una subvención normativa, mediante fórmula y como subvención global con plena libertad de adscripción. Para gastos corrientes la financiación se completa con subvención para gastos de inversión en planes plurianuales. Crece la difusión de los "contratos-programa".

40. Los sistemas universitarios con elevado número de instituciones y financiación mediante fórmula se apoyan en la existencia de instituciones intermedia a modo de "buffer", mientras en un sistema descentralizado no hay espacio para intermediarios.

41. El modelo de financiación debe cubrir objetivos y garantizar la suficiencia financiera, permitiendo cumplir los objetivos y protegiéndolas de las presiones políticas. Debe ser transparente, fijar sus prioridades e incentivar el logro de sus objetivos.

42. En los años noventa, se elaboró el Informe de Financiación de 1995. La Comunidad Valenciana usó fórmulas de subvención, Canarias y Cataluña contratosprograma y la Comunidad de Madrid desarrollo un plan plurianual. 
43. En España la subvención pública podría articularse en torno a una fórmula referenciada a los costes corrientes y como elementos básicos: el número equivalente de estudiantes y los costes estándar. El concepto de estudiantes a tiempo completo se estimará dividiendo el número total de créditos por 65 o 70. Se debe eliminar los incentivos a la elevación desmesurada de la carga docente estableciendo un número máximo de créditos financiables y de asignaturas optativas financiables. (Apartado III-63 del Informe de Financiación 1995). La fórmula lineal en función al número de estudiantes no es eficiente. Se recomiendan factores que incentiven el ajuste de la demanda, titulados de calidad y economías de escala.

44. Dado el sistema actual el modelo deberá considerar las preferencias en la preinscripción primando las plazas de primera opción, concediendo financiación extra por estudiante admitido en primera opción. Los nuevos modelos pueden recoger las economías de escala introduciendo un elemento fijo en la subvención. Se debe incluir incentivos a la salida de graduados, titulados competentes, estimulando un aumento de la productividad y realizar un seguimiento de la carrera profesional de sus titulados. El modelo de financiación para gastos corrientes se completaría con una subvención para gastos de inversión, requiriendo la coordinación de los gobiernos de las CC. AA. Prestar atención especial a las necesidades de equipamiento de las universidades.

45. Financiar a grupos y actividades de especial calidad fomentando la competencia, incluir financiación para potenciar innovaciones docentes y actividades de mejora de la calidad de la enseñanza. Ejemplos de programas específicos:

Subvencionar la investigación de los departamentos universitarios. Consolidar programas de doctorado destacados por su calidad. 
Programas competitivos para incentivar la innovación en las actividades docentes.

Vincular las convocatorias de becas de formación postgrado y los programas competitivos. En la concesión de becas evaluar la viabilidad de los programas de doctorado. Para los programas profesionales ofrecer ayuda financiera mediante préstamos.

46. Muchos de los programas competitivos deben afectar al conjunto del sistema universitario español. En los países de estructura federal se impulsen programas federales de financiación.

47. La adaptación de las instituciones de enseñanza superior a los objetivos fijados podría instrumentalizarse mediante los contratos-programas.

4.- El reparto de responsabilidades en la financiación de la educación superior

48. UNESCO, París, octubre 1998. "La financiación de la educación superior exige recursos públicos y privados. El papel del Estado permanece esencial a este respecto". El análisis de la financiación en España aconseja proponer cambios progresivos y no traumáticos. La finalidad última es lograr la suficiencia financiera e incrementar la responsabilidad de las universidades frente a los gobiernos y frente a los estudiantes. La situación actual es:

El gasto total en educación superior representa el 1,1\% del PIB (la media de la OCDE es del $1,6 \%)$

El gasto anual por estudiante es aproximadamente de 5.000 dólares (el 60\% de la media de la OCDE). 
El sistema universitario español debería aumentar su gasto por estudiante. Ello requiere aumentar el gasto total (público y privado) en enseñanza superior. Este objetivo puede alcanzarse a medio plazo, el cual no ha de exceder de 10 años.

En relación con el reparto del esfuerzo entre gasto público y gasto privado, conviene que recordar que:

La proporción de los recursos públicos en España es del 76\% (la media OCDE es del $86 \%)$, la aportación privada es del $24 \%$.

La subvención a estudiantes y familias, por diferentes conceptos, representa en España el 0,06\% del PIB (la media europea es del 0,25\%).

El importe medio de las becas en España era de 109 ECUS por mes en España (1996). En España el porcentaje de familias que se acogen a becas es el 17,3\%.

Las principales partidas que componen la estructura de la financiación de las universidades públicas y privadas:

La subvención directa a las universidades públicas, que supone un 0,8\% del PIB.

Los pagos privados a las instituciones educativas públicas y privadas $(0,25 \%$ del PIB).

Los subsidios o ayudas públicas a las familias.

Otras categorías de gastos de escasa importancia. 
El sistema de financiación debe mantener un modelo mixto (público-privado). Es recomendable, por tanto, que la participación privada a través de las tasas, por lo menos, no supere el porcentaje actual en el conjunto de los fondos de financiación de las instituciones universitarias.

Se aconseja destinar una buena parte del aumento de los recursos públicos a la enseñanza superior incrementando las ayudas a los estudiantes y a sus familias; y aumentando el peso de las subvenciones a los estudiantes y las familias en relación con las subvenciones directas y facilitando la movilidad a los estudiantes.

El crecimiento de la población universitaria en el futuro es de estimación incierta. Se ha supuesto que no habrá crecimiento.

Aumentar sustancialmente el número de estudiantes que reciben becas, llegando al 40\% para los estudiantes de los primeros cursos. Las becas cubrirán un $10 \%$ de los estudiantes de últimos cursos, en los cuales se dará prioridad al sistema de préstamos-renta. La cuantía de las ayudas deberá incrementar hasta llegar a las 400.000 pesetas. Una parte de la financiación de las universidades, deberán conseguirla a través de la atracción de estudiantes con o sin becas.

Poner en marcha un programa piloto de préstamo-renta orientado a los estudiantes de últimos cursos ya que este colectivo tiene mayores expectativas de poder hacer frente a la devolución. El programa debería ir dirigido a cubrir a un 30\% de los estudiantes de los últimos cursos. A largo plazo los retornos y los préstamos deberían equilibrarse. Su devolución dependería, en gran medida, de la idoneidad de los estudios cursados con relación a las oportunidades de ejercicio profesional. Antes de generalizar el sistema de préstamos-renta parece conveniente introducir un programa experimental de movilidad. 
La subvención directa a las universidades deberá repartirse entre convocatorias, contratos-programas y subvenciones nominativos con criterios objetivos, aumentar el peso relativo de la financiación mediante convocatorias y contratos-programas respecto al de las subvenciones.

Simulación de la evolución en las fuentes de financiación de la educación superior:

Subida del gasto de 200.000 pesetas por estudiante.

El volumen total en estudiantes se sitúa en torno al 1.785.000.

El porcentaje del gasto que correspondería:

Las Administraciones Públicas el 80\%.

El sector privado el $20 \%$.

Las cifras propuestas de crecimiento darían lugar a un aumento decenal de 357 millones de pesetas, hasta conseguir el 1,52\% del PIB al final del período. Se distribuiría de la siguiente forma:

Un crecimiento de 139.000 millones de pesetas del gasto público destinado alas instituciones educativas.

Un crecimiento de dicho gasto privado de 43.500 millones de pesetas.

El mayor crecimiento correspondería a la ayuda pública a los estudiantes. 
En total se pasaría pues a un gasto en ayudas a estudiantes universitarios en torno a los 174 millones de pesetas.

\section{VI.- PERSONAL DE LA UNIVERSIDAD}

\section{(A) RESUMEN Y PROPUESTAS}

1.- Gestión de recursos humanos

\section{1.- Un nuevo entorno.}

(1) La calidad de una institución de educación superior depende en gran parte de sus miembros. Por tanto, los profundos cambios a abordar en la U. afectarán también al PDI y al PAS.

(2) Por ejemplo, en la necesidad de formación permanente (TIC) o de movilidad.

(3) En particular, es necesaria una planificación estratégica de las necesidades de personal, que requiere un diseño de su plantilla. El principal problema es que debe partirse de una situación ya existente: hay que referirse más a las necesidades del entorno que a las internas de reproducción y promoción.

1.2.- Planificación de necesidades.

(4) Es necesario flexibilizar las figuras disponibles (funcionarias o contractuales) así como sus categorías.

La política de personal deberá tener en cuenta los salarios y perfiles exteriores (quizá habrá que recurrir a tiempos parciales), la reserva de reclutamiento, las futuras 
jubilaciones y otros muchos factores externos (competencia, demanda,...) e internos (características académicas, territoriales, estructura,...) por lo que el proceso no es lineal y general, sino iterativo y particular.

(5) El planeamiento debe recoger el número de plazas de los distintos niveles, su dedicación y finalidad, y las prioridades. Y análogamente para PNN.

Se puede empezar por planes parciales de cada unidad, aunque éstas suelen tender más a la improvisación que la planificación. Además su sistema de toma de decisiones prima la participación sobre la eficacia y la racionalidad, y las intervenciones externas son problemáticos (por desconocedoras y mal acogidas). La creciente cultura de la evaluación hará cambiar estas actitudes.

(6) Internamente, la planificación debe hacerse tanto "de abajo a arriba" cm al revés. Debe negociarse con las Administraciones Públicas, teniendo en cuenta la diversidad, y tendiendo a negociaciones globales de plazas, en vez de individualizadas.

Contra la visión usual de la U. como dinamizadora de su auto-reforma, se tiende hacia Consejos de Administración externos. Conviene que sean las Administraciones Públicas quienes estimulen la planificación estratégica de la que se derive la plantilla.

(7) Hay que tener en cuenta la percepción del propio personal, para no desmotivarlo.

(8) La planificación de personal ha de prever el número, pero sobre todo el perfil de los puestos. La "gestión por competencias" de personal en la U. ha de ajustarse a los activos disponible o incorporables, y las competencias que cada persona ofrece (no sólo conocimientos y destrezas sino también motivación, carácter, autoestima, actitudes, valores). Igualmente, ha de establecer para cada puesto no sólo el perfil genérico sino las competencias requeridas (tanto "de umbral" como 
"diferenciadoras"): definición del puesto de trabajo, tareas y actividades, formación y experiencia, conocimientos, destrezas. Entre las competencias evaluables hay que incluir también las difíciles de obtener: comunicación, dirección, liderazgo,...

Objetivos de todos ello son: configuración de equipos de trabajo, cultura de la mensurabilidad, gestión del cambio.

(9) Debe complementarse con una evaluación integral que se desarrolle y perciba como un instrumento para mejorar.

(10) Probablemente la planificación resultante de necesidades de personal será incompleta, imperfecta e inadecuada; pero será más útil que no tenerla.

\section{2.- Profesorado.}

\section{1.- Problemas.}

(11) Entre las dificultades para elaborar la plantilla de PDI se señalan: compatibilizar investigación con docencia (sobre todo en primeros ciclos), degradación de la función docente, falta de visión global y de compromiso con la institución,... En este sentido, las individualidades deben aunar sus esfuerzos.

(12) El proceso de inducción pretende que el personal de comprometa con la institución.

Las U. tendrán que afrontar dos cambios: curriculares, y de organización. En efecto, la escasa eficiencia resulta para unos de le escasa financiación, y para otros de la propia estructura que da la última palabra a su propio personal, no suficientemente comprometido con la colectividad. 
La necesidad de reforzar este carácter de colectividad debe manifestarse también en la selección de PDI: no del genéricamente mejor, sino del más adecuado; de ahí la necesidad de un perfil (definido por la institución, no por el propio departamento).

(13) Un problema recurrente ha sido y es el del PNN.

Es difícil analizar los modelos de plantilla en otros países, ya que sólo se ha planteado desde los 80 , cuando han aumentado los estudiantes y han disminuido los recursos.

(14) Hay que reafirmar la indivisibilidad entre docencia e investigación, con lo que el más adecuado quizá no sea el mejor investigador. Habrá que contar con una figura preferentemente docente y otra preferente investigadora, cuidando el equilibrio entre ambas. Por ejemplo, en enseñanzas más profesionalistas se necesitan los primeros.

(15) Algunas exigencias son:

correcta ratio estudiantes/profesor

remuneración adecuada

justificar la dedicación a la investigación

recobrar los asociados LRU

2.2.- Figuras de personal académico.

(16) En cuanto a figuras de profesorado se recomienda:

CU, TU: mantenerlas como ahora

CEU, TEU: no se considera un tema relevante, pero se sugiere suprimirlas a medio plazo (negociar su futuro con ellos y también con los sindicatos) 
Asociados: volver a la LRU; se recomienda el contrato directo en vez del concurso; que incluso puedan ser PAS

Ayudantes: mantenerla como en la LRU, enfatizando que no tiene plaza asegurada; concretamente se sugiere 3 años improrrogables como "Ayudante" (no doctor) o "Ayudante doctor" para capacitación pedagógica

Visitante: incentivarla

Emérito: para evitar desviaciones, que lo decida la U. sin participar el departamento

(17) Hay que evitar malos usos de las anteriores figuras y paliar desajustes entre necesidades docentes e investigadoras

(18) Por ello se proponen dos nuevas figuras contratadas:

Titular Docente (TD): para docencia de primer y segundo ciclo; desde Ayudante de ambos tipos

Titular Investigador (TI): para investigación y docencia de doctorado; desde Ayudante doctor, o doctores en general

3.- Carrera académica y procedimiento de selección

\section{1.- Carrera académica}

(19) La carrera académica es la progresiva capacitación y adquisición de competencias (conocimientos, habilidades, aptitudes). No excluiría otros accesos.

En la actualidad no la hay, por causas difíciles de precisar.

(20) Ha de establecer figuras iniciales y las exigencias de promoción, evitando acumulación de PNN como las habidas. Ha de encauzar la promoción, dejando claro 
que la formación no garantiza plaza. Ha de relacionarse con la planificación y previsión de plantilla, e incluso con las posibles salidas hacia el exterior.

Se señala una fuerte demanda por jubilaciones hacia 2005-2010, lo que debería aprovecharse para mejorar el tercer ciclo, y para que la formación empiece tras la tesis.

(21) Hay experiencias interesantes en Francia (monitores), Holanda (asistentes de investigación),...

(22) En España se empieza con el doctorado (también es necesario el tercer ciclo), que no es una iniciación a la docencia. Hay que completarla con formación metodológica, uso de TIC,,..

(23) Una vía sería Ayudante y después Titular Docente.

Otra vía sería el doctorado (becas,...), y después Ayudante-doctor adscrito a un centro acreditado por la Agencia de Acreditación, para ser al final reconocido como "investigador". Entonces podría acceder a Titular Investigador, e incluso a TU, así como a Titular Docente.

(24) Por tanto, habría tres tipos de carrera (sólo docente, sólo investigadora, o mixta) para garantizar suficiente flexibilidad.

Habría otras exigencias específicas: en medicina, enseñanzas profesionalistas,...

(25) Se podría acceder a TD y TI desde otras vías, exigiendo el doctorado para TI. 
(26) Hay que garantizar y promover la formación permanente del personal universitario: toma de conciencia, méritos para evaluación,...

Por ejemplo, se debería garantizar el año sabático, fomentar la movilidad y la internacionalización, y valorar la formación continua en la selección de PDI

\section{2.- Incentivos y motivaciones.}

(27) A pesar de la mejora que supuso los quinquenios y sexenios, las retribuciones siguen siendo bajas (el art.11 afecta a una minoría) y poco estimulantes, por ejemplo, para la productividad. Se sugiere establecer incentivos adicionales, como premios consolidables por evaluación externa positiva; también suprimir el tope de sexenios.

(28) No entra a fondo en la mejora las condiciones de trabajo, citando que a menudo esa es la recompensa por una evaluación positiva, o en sentido contrario los efectos negativos de la escasez de PAS.

En este contexto de deficientes retribuciones y condiciones de trabajo, hay que destacar el trabajo anónimo de muchos integrantes de la U., poco reconocido. Para mantener esta motivación hay que eliminar algunas fuentes externas de insatisfacción, más que crear nuevas recompensas externas o sanciones.

El síndrome de "burn out" es muy negativo para la institución, pero de hecho una parte importante del PDI padece estrés por exceso de trabajo y falta de tiempo.

\section{3.- Selección del profesorado}

(29) Los mecanismos de selección perderán trascendencia si se asegura una buena formación. 
Con todo, habría que revisar el peso excesivo de la investigación (que quizá estaba justificado en los 80), planteando una mayor adecuación a las tareas a desarrollar, lo que en ciertos casos significará enfatizar los aspectos docentes.

Nuevamente hay que insistir en la movilidad.

(30) En la alternativa entre regular o no la selección, hay que garantizar: que se seleccione al más adecuado para la función precisa; la autonomía universitaria.

El procedimiento descansaría en tres pilares:

planteamiento previo de las funciones a cubrir informe de expertos, no necesariamente del área

decisión de la U. (global, no restringida al propio grupo; quizá un Comité Académico de la U.)

Estos principios se aplicarían a todas las selecciones

Los contratos podrían ser indefinidos para TU y TI.

(31) No parece que en España el modelo de habilitación sea el más adecuado. Se sugiere un método que de hecho ya se usa en el sector público.

Se dividiría en dos fases la selección de funcionarios:

preselección por un comité de especialistas externos, ordenándolos separadamente para cada aspecto a considerar

decisión por personas de la propia U., como mejor conocedora de las necesidades a cubrir 
Sería mejor que el mero endurecimiento de las pruebas, siempre que se garantice una formación contrastada.

\section{4.- Personal de administración y servicios}

Con respecto al Personal de Administración y Servicios el informe señala la diversificación existente en la actualidad sobre la presencia de los colectivos en las diferentes Universidades españolas así como un buen análisis de las diferentes características tanto del Personal Laboral como del Personal Funcionario.

(32) El informe establece la conveniencia de adoptar medidas de homologación y homogeneización de las plantillas dentro de ámbitos de mesas sectoriales y define con bastante exactitud las competencias de los dos colectivos dentro de las Universidades.

(33) La pieza clave en materias de plantillas son las RPT, definiéndolas en la actualidad como demasiado rígidas y lejanas a los principios de racionalidad, economía y eficiencia que serían exigibles para las necesidades actuales de las Universidades. Recomienda por tanto la creación de una RPT tipo, dentro del ámbito de las Comunidades autónomas, que garantice la homogeneidad de las diferentes plantillas entre las Universidades de ese ámbito.

(34) El informe plantea la necesidad de asunción de competencias por parte de las Comunidades Autónomas con respecto al Personal de administración y Servicios, recomendado la creación de una Mesa Unitaria para el PAS dentro de la Mesa Sectorial de Universidad, igualmente plantea la necesidad de que estos acuerdos de carácter autonómico sean de obligado cumplimiento tanto para Las Universidades como para los órganos de representación Unitarios, por ultimo hace mención a la 
recomendación de crear un convenio multilateral que facilite la movilidad del PAS entre las diferentes Universidades.

(35) Analiza la estructura retributiva del Personal de Administración y Servicios, planteando el actual sistema como un sistema rígido, ineficaz y extremadamente complejo, añade el informe la falta de conceptos retributivos por incentivos y la perversión del concepto de productividad en el caso del Personal Funcionario.

El informe destaca como única vía posible para fijar políticas universitarias desde la cúpula funcionarial, el actual sistema de puestos de libre designación y añade en sus conclusiones el poco desarrollo de una autentica carrera administrativa dentro del ámbito universitario.

(36) Se aborda la equiparación en cuanto al desarrollo de la formación continuada de las mismas políticas que recomienda en apartados anteriores para el Personal Docente.

Recomienda por lo tanto la promoción de consorcios entre Universidades, inicialmente de la misma Comunidad Autónoma, para programar adecuadamente la formación del Personal de Administración y Servicios.

(B) VALORACION Y SUGERENCIAS

1.- Gestión de recursos humanos

Es positiva la insistencia en la necesidad de planificar la necesidad de personal, y por lo tanto la plantilla. 
Se deslizan valoraciones muy negativas sobre la capacidad de autogobierno participativo (5) y de autoreforma (6) de la U., dando por hecho que, al contrario, la intervención de externos o de la Administración resultaría provechosa. No compartimos esas valoraciones, que en cualquier caso corresponden al cap.VIII. en particular, parece contradictorio con la insistencia en mantener la motivación y la corresponsabilidad del personal.

Interesante la "gestión por competencias", aunque se reconoce que los frutos pueden ser decepcionantes en la U. Es dudoso que una planificación de personal "incompleta, imperfecta e inadecuada" pueda ser más útil que no tenerla: cuando se tiene, hay la tentación de usarla.

CONTINUARÁ... 